\title{
Dark Energy And Cyclic Universe From Arnowitt-Deser-Misner Renormalizable Group Approach
}

\author{
El-Nabulsi Ahmad Rami* \\ Department of Nuclear and Energy Engineering, Cheju National University, Ara-dong 1, Jeju 690-756, South Korea
}

(Received on 30 December, 2008)

\begin{abstract}
We discuss the generic features of the accelerated universe from Arnowitt-Deser-Misner renormalizable group approach applied for gravity models with variable gravitational coupling constant and cosmological constant. The universe undergoes an endless sequence of cosmic eras which starts inflating after a Big Bang and end contracting to a Big Crunch dominated by phantom energy. Presently, the universe found to be presently accelerating in time and is dominated by dark energy. Some important features and numerical results are revealed and discussed in some details.
\end{abstract}

Keywords: Ddark energy; Phantom energy; ADM formalism, accelerated expansion; Cyclic universe; Bekenstein entropy

Recent astronomical observation on the Cosmic Microwave Background (CMB) Anisotropy [1] and Supernova Type Ia (SNIa) [2] suggest that our Universe undergoes a phase of accelerated expansion tending to a flat de-Sitter space-time as predicted by inflation theory [3] and in accord with recent findings of BOOMERANG experiments [4]. In fact $70 \%$ of the total density results from a mysterious and unknown vacuum energy density or an effective equivalent, and weakly interacting dark matter [5-9]. The nature of the dark energy component represents one of the most profound and important tasks for modern cosmology, astrophysics and theoretical physics. This dark energy was important in the past as it is now. It might have played a part in limiting the formation of largest gravitationally bound structures. Several candidates to represent and explain dark energy have been postulated and confronted with observations: the $\Lambda \mathrm{CDM}$ ( $\Lambda$-Cold-Dark-Matter) matter [10] consisting a mixture of cosmological constant $\Lambda$ and cold dark matter (CDM) or WIMPS composed of weakly interacting massive particles which must be relics of a grand unified phase of the Universe, quintessence with a very shallow many-forms potential [11], K-essence [12], viscous fluid [13], Chaplygin gas [14,15], Generalized Chaplygin gas model (GCGM) which mimics both dark matter and dark energy [16,17], Brans-Dicke (BD) pressureless solutions [18] and so on.

Most of these theories are problematic and faced many difficulties. For example, within the framework of the $\Lambda \mathrm{CDM}$ cosmological scenario, the vacuum energy is set to be constant with time while the matter energy density is a decreasing quantity, their ratio must be set to a specific infinitesimally small value $10^{-120}$ in the early Universe so as to nearly coincide today, i.e. a huge vacuum energy which by about 120 orders of magnitude exceeds the experimental limit. This is the famous "cosmic coincidence" problem (CCP). Present astronomical observations indicate that the matter contribution $\Omega_{m}$ to the present universe is such that $0.1<\Omega_{m}<0.4$. One can model the dark energy by a cosmological constant (or a vacuum decaying energy). One interesting possibility is that the cosmological constant may be effective, and indeed could be varying with cosmic time. As we strongly believe that the effective cosmological constant is very close to zero, the effective lambda may be identified to a variable dynamic degree of freedom so that in an expanding it relaxes to zero. Some interesting phenomenological models include $\Lambda \propto H^{2}, \Lambda \propto \ddot{a} / a$,

*Electronic address: nabulsiahmadrami@yahoo.fr
$\Lambda \propto 8 \pi G \rho, \Lambda \propto R$, etc. [19] $H, a(t), \rho$ and $R$ are respectively the Hubble parameter, the scale factor, the matter density and the Ricci curvature scalar. They are in fact appealing theories, not excluded from cosmological, astrophysical and high energy physics literatures.

Moreover, there have been several successful attempts at building alternative theories of gravities which could be responsible for the current accelerated expansion. These scalartensor theories of gravity revealed interesting consequences and have potential to provide a linkage between the accelerated expansion of the universe and fundamental physics although the acceleration cannot be explained by the standard model of particle physics and classical general relativity. Some nice alternatives theories include the string-inspired dilaton gravities, a time-varying energy density, dilaton from string theory, supersymmetric exotic particles, massive neutrinos, phantom energy and the higher derivative theories with an additional quadratic scalar curvature [20], which may mimic the effects of DE on the Hubble flow.

In reality, cosmological models with $G$ and $\Lambda$ treated as spacetime functions have acquired greater relevance after the recent evidence in favour of the Einstein theory being nonperturbatively renormalizable in the Weinberg sense [21]. Within this structure, the basic ingredient to support $G$ and $\Lambda$ to the role of spacetime functions is the renormalization group (RG) progress, a standard tool in elementary particle physics in order to insert the leading quantum corrections to the Born approximation of a scattering cross section. The RG-improved of the Einstein's field equations can therefore be obtained in a similar way by simply replacing $G \rightarrow G(k)$ and $\Lambda \rightarrow \Lambda(k)$, $k$ being the running mass scale identified in general with the Hubble parameter or the inverse of cosmological time in the Friedmann-Robertson-Walker (FRW) spacetime. Within this approach, it has been shown that the RG framework provide a possible solution to many of the fundamental problems in cosmology in particular the horizon and flatness problem of standard FRW cosmology without the need of the inflationary mechanism. They represent also a promising model of dark energy in the late universe. Further, when applied the Arnowitt-Deser-Misner (ADM) Lagrangian formalism to such a class of gravitational models, in particular to gravity coupled to a massless self-interacting scalar field in the FRW spacetime, it has been revealed that the density of matter decays like $\rho \propto \dot{G}^{2} / G^{3}$, which is considered as one of the main features of the model. The basic idea of this approach known as ArnowittDeser-Misner (ADM) is similar to the renormalization group based derivation of the Uehling correction to the Coulomb po- 
tential in massless QED.

The aim of this short communication is to extend further the investigations of this decaying law to obtain general solutions of the cosmological equations and to check its consistency with the available observational data via the neoclassical tests. No scalar field will be included in the theory. Further, we adopt the phenomenological law $\Lambda \propto H^{2}$ as it may explain why the effective cosmological constant is reduced from a large value at early times to a sufficiently small value at late times in consistent with observational upper limit. Special attention is paid for scaling solutions which could be important building blocks in constructing the models of dark energy. We expect that this model may have many interesting features for producing in a natural way an epoch of accelerated expansion of the universe. The author may be refereed to our recent works to explore the important role of scaling solutions in accelerating cosmology [20].

We really believe inflation theory in all its aspects predicting $\Omega_{k}=-0.015_{-0.016}^{+0.020}$ (within a $2 \%$ margin of error consistent with nearly scale-free, Gaussian, adiabatic primordial density perturbations (the kind predicted by the inflationary universe scenario and accelerating in time)). For this, we consider the standard four-dimensional flat homogeneous Friedmann-Robertson-Walker universe described by the metric $d s^{2}=-d t^{2}+a^{2}(t) \sum_{i=1}^{3} d x_{i}^{2}$ where $a(t)$ is the scale factor of the universe. For the prefect fluid distribution the Einstein field equations with the effective cosmological constant and gravitational constant may be written as $R_{\mu v}-(1 / 2) g_{\mu v} R+$ $\Lambda_{\text {effective }} g_{\mu v}=-8 \pi G T_{\mu v}$ where $T_{\mu v}=-\left[(p+\rho) u_{\mu} u_{v}+p g_{\mu v}\right]$ is the stress-energy tensor. $p$ and $\rho$ are the pressure and the density of the perfect fluid.

The variations of the gravitational constant, the cosmological constant and the fluid density in cosmological time will in turn influences the expansion of the universe, through the generalization of the Friedmann equation which takes the standard form $3 H^{2}=8 \pi G(t) \rho(t)+\Lambda(t)$. Further, The Bianchi identity $\left[R^{\mu \nu}-1 / 2\left(g^{\mu \nu} R\right)\right]_{; \mu}=-\left[8 \pi G T^{\mu \nu}+\Lambda g^{\mu \nu}\right]_{; \mu}=0$ leads to the useful equations: $\dot{\rho}+3 H(p+\rho)=0$ and $\dot{\Lambda}+8 \pi \dot{G} \rho=0$. Adopting in what follows the equation of state $p=w \rho, w$ is the equation of state parameter, the first differential equation is written like: $\dot{\rho}+3 H(w+1) \rho=0$. In reality, we allow the gravitational coupling to vary with time in order to retain the energy conservation, i.e. the variation of lambda is cancelled by the variation of $\kappa^{2}$. Although the proper way is to look for a field phenomenological framework for the variation of the gravitational coupling constant and the cosmological constant, nevertheless the present approach could be considered as a limiting case of some covariant theory yet to be discovered. Brans-Dicke scalar theory is one prototype of these theories but as it is well-known, this theory allows only a decreasing gravitational constant with the cosmological time.

We are mainly interested in power-law solutions which can exist in such a model. To explore the scaling behaviors, we assume that the scale factor and the gravitational coupling constant in the theory described evolve respectively as $a=a_{0} t^{m}$ and $G=G_{0} t^{p} ; m$ and $p$ are constants; $a_{0}$ and $G_{0}$ are the values of the parameters at the present epoch assumed equal to unity for simplicity. For a matter density decaying like $\rho=$ $3 \beta \dot{G}^{2} / 8 \pi G^{3}=3 p^{2} t^{-2-p} / 8 \pi G_{0}, \beta$ is a constant assumed for mathematical convenience to be close to unity and $\Lambda=3 \gamma H^{2}$, $\gamma$ is a positive constant.

For a flat universe, the Friedmann equation gives $p^{2}=$ $m^{2}(1-\gamma)$ while the continuity equations yield $2+p=3 m(w+$ $1), w \neq-1$ and $p^{3}=2 \gamma m^{2}$ from one deduces that $p=$ $2 \gamma /(1-\gamma)$ and $m^{2}=4 \gamma^{2} /(1-\gamma)^{3}$. Notice that for $\gamma=1$, we find the standard case in which $\Lambda=3 H^{2}, p=0$ and therefore the gravitational coupling is constant while the matter density decays like $\propto \propto t^{-2}$ and the scale factor behaves like $a(t) \propto t^{2 / 3(w+1)}$. That is, due to dark energy, the expansion of the universe $a(t) \propto t^{(2+p) / 3(w+1)}$. To experience accelerated expansion, $\ddot{a}>0$, which requires $2+p>3(w+1)$. When the dark energy dominates neglecting the matter, the scale factor evolves exponentially.

Present observations indicate that the universe is accelerating in time, i.e. $m>1$ and that the matter contribution to the present universe is such that $0.1<\Omega_{m}=p^{2} / m^{2}=1-\gamma<0.4$. Here $\Omega_{m}=\rho / \rho_{c}$ where $\rho_{c}=3 H^{2} / 8 \pi G$ is the critical density of the universe. Consequently one may easily deduce that $0.6<\gamma<0.9$ or $1.8 H^{2}<\Lambda<2.7 H^{2}$ and $0.316<p / m<0.632$. As a simple numerical example, we choose $\gamma=0.75$ for which one finds easily: $p=6, m=12, \Lambda=2.25 H^{2} \propto t^{-2}, \rho \propto t^{-8}$ and $G \propto t^{6}$. In fact, the present day variation of the gravitational coupling constant $\dot{G}_{0} / G_{0}=(p / m) H_{0}=0.5 H_{0}$ which for $h=0.7_{-0.017}^{+0.016}$ where $h \equiv H_{0} / 100 \mathrm{~km} / \mathrm{s} /$ Mpcis allowable value if compared with the results of observations. [20,21] The accelerated expansion is possible only when $-1<w<-0.77$.

The universe is therefore dominated by dark energy and is accelerating with time. It is noticeable that the phantom barrier $w=-1$ is not allowed and therefore is not crossed. The matter contribution to density parameter of the universe is $\Omega_{m} \approx 0.25$ and consequently the vacuum contribution $\Omega_{\Lambda}=1-\Omega_{m} \approx 0.75$ (about $75 \%$ is dark energy) in agreement with observational limit, in particular the parameters derived from WAMP, the "apparent magnitude- redshift" neoclassical test, realized for SNIa, the power spectrum of CMB temperature fluctuations, obtained in ground based, stratospheric and cosmic experiments and other projects data: [22] $\Omega_{\Lambda}=$ $0.745_{-0.017}^{+0,017}, w=-0.915_{-0.051}^{+0,051}$, and $\Omega_{m}=0.255_{-0.017}^{+0,017}$ (the best fit values and their $1 \sigma$-confidence intervals). Further, one find $H_{0} t_{0}=12$ in contrast to the standard model which predicts a shorter age of the universe. According to modern astronomical data the dust matter consists of cold dark matter with $\Omega_{C D M} \approx 0.21$ and usual baryons $\Omega_{B} \approx 0.05$. In our framework, $\Omega_{\Lambda}>\Omega_{C D M}>\Omega_{B}$ similar to the one obtained in Quintessence Cold Dark Matter (QCDM) model.

As $p$ and $m$ change during the history of time, one then expects that $w$ will change in time. For the matter-dominated universe, $w=0$ and hence one finds easily $\gamma_{+}=0.28$ (the negative value is not allowed for a universe dominated by a positive cosmological constant), $p=0.77$ and $m=0.916$. For a radiation-dominated universe, $w=1 / 3$ and therefore $\gamma_{+}=$ $0.22, p=0.56$ and $m=0.638$. It can be easily seen that during the radiation-dominated epoch of time, the scale factor grows as $a \propto t^{0.638}$ (decelerated expansion) and later during the matter-dominated epoch, the expansion starts increasing like $a \propto t^{0.916}$ until the time the dark energy accelerates the expansion of the universe.

We summarize in the following table for different epochs of time; in particular for the phantom- 
dominated epoch, we choose the numerical values $w=[-0.77,-6 / 5,-3 / 2,-2,-5 / 2,-3,-10]$. At present, to meet the observational result, we choose $w \approx-0.77$. Further, the late-time epoch dominated by dark energy is characterized by $\gamma=0.75$, a numerical value chosen by hand.

TABLE 1

\begin{tabular}{|l|l|l|l|l|l|l|l|l|l|}
\hline$w=\frac{2+p}{3 m}-1$ & $\gamma$ & $p$ & $m$ & $\Lambda=3 \gamma H^{2}$ & $\rho \propto t^{-2-p}$ & $G \propto t^{p}$ & $a \propto t^{m}$ & $\Omega_{m}$ & $\Omega_{\Lambda}$ \\
\hline $\begin{array}{l}\text { Phantom Energy Dominance } \\
w=-10 ; 2+p=-27 m\end{array}$ & 0.036 & 0.0746 & -0.076 & $0.108 H^{2}$ & $t^{-2.0746}$ & $t^{0.0746}$ & $t^{-0.0768}$ & 0.94 & 0.06 \\
\hline $\begin{array}{l}\text { Phantom Energy Dominance } \\
w=-3 ; 2+p=-6 m\end{array}$ & 0.153 & 0.36 & -0.39 & $0.459 H^{2}$ & $t^{-2.36}$ & $t^{0.36}$ & $t^{-0.39}$ & 0.85 & 0.15 \\
\hline $\begin{array}{l}\text { Phantom Energy Dominance } \\
w=-5 / 2 ; 2+p=-9 m / 2\end{array}$ & 0.198 & 0.49 & -0.55 & $0.594 H^{2}$ & $t^{-2.49}$ & $t^{0.49}$ & $t^{-0.55}$ & 0.8 & 0.2 \\
\hline $\begin{array}{l}\text { Phantom Energy Dominance } \\
w=-2 ; 2+p=-3 m\end{array}$ & 0.28 & 0.77 & -0.92 & $0.84 H^{2}$ & $t^{-2.77}$ & $t^{0.77}$ & $t^{-0.923}$ & 0.7 & 0.3 \\
\hline $\begin{array}{l}\text { Phantom Energy Dominance } \\
w=-1.5 ; 2+p=-3 m / 2\end{array}$ & 0.48 & 1.84 & -2.56 & $1.44 H^{2}$ & $t^{-3.84}$ & $t^{1.84}$ & $t^{-2.56}$ & 0.51 & 0.49 \\
\hline $\begin{array}{l}\text { Phantom Energy Dominance } \\
w=-1.2 ; 2+p=-3 m / 5\end{array}$ & 0.24 & 0.63 & 4.38 & $0.72 H^{2}$ & $t^{-2.63}$ & $t^{0.63}$ & $t^{-4.38}$ & 0.75 & 0.25 \\
\hline $\begin{array}{l}\text { Dark Energy Dominance } \\
w \approx-0.77 ; 2+p=0.69 m\end{array}$ & 0.75 & 6 & 12 & $2.1 H^{2}$ & $t^{-8}$ & $t^{6}$ & $t^{12}$ & 0.25 & 0.75 \\
\hline $\begin{array}{l}\text { Matter-Dominated Epoch } \\
w=0 ; 2+p=3 m\end{array}$ & 0.28 & 0.77 & 0.916 & $0.84 H^{2}$ & $t^{-2.77}$ & $t^{0.77}$ & $t^{0.916}$ & 0.7 & 0.3 \\
\hline $\begin{array}{l}\text { Radiation-Dominated Epoch } \\
w=1 / 3 ; 2+p=4 m\end{array}$ & 0.22 & 0.56 & 0.638 & $0.66 H^{2}$ & $t^{-2.56}$ & $t^{0.56}$ & $t^{0.638}$ & 0.77 & 0.23 \\
\hline $\begin{array}{l}\text { Stiff Fluid-Era } \\
w=1 ; 2+p=6 m\end{array}$ & 0.153 & 0.36 & 0.39 & $0.459 H^{2}$ & $t^{-2.36}$ & $t^{0.36}$ & $t^{0.39}$ & 0.85 & 0.14 \\
\hline
\end{tabular}

It is noteworthy that an inflationary solution is obtained for $w=-1$, i.e. from which one obtains easily the standard inflationary de-Sitter solution $a(t) \propto e^{H t}$. The solutions demonstrate that the universe starts with an exponential expansion but with infinite deceleration parameter $q=-\ddot{a} a / \dot{a}^{2}=$ $(1-m) / m \rightarrow \infty$ and infinite equation of state parameter $w \rightarrow$ $\infty$. In the process of the cosmological evolution, it passes though the stiff fluid era $(w=1)$, the radiation-dominated era $(w=1 / 3)$ and the matter-dominated era $(w=0)$. After that, the universe encounters a turning point when $w=-0.77$ and starts accelerating with time. If a transition from dark energy to phantom energy occurs, the universe enters asymptotically a collapsing period of time and it may easily check that for $w<<0, a(t) \rightarrow$ constant value (static universe) dominated by matter and therefore, a cyclic cosmology may occurs (the universe enters a new inflationary era). When the phantom barrier $w=-1$ is crossed, the expansion starts contracting.

Therefore, the evolution of the cosmological parameters is able to phantomize $(w<-1)$ the model unless the universe is decelerating (collapsing) with time and became dominated by matter. In other words, the universe undergoes an endless sequence of cosmic epochs each beginning with a 'Big Bang' and ending in a 'phantom-crunch'. Each cycle starts with inflation followed by contraction, then acceleration and finally a transition from acceleration to a collapsing or contracting phase. We argue that we are living in the stage where the dark energy plays the role of the expansion of the universe. Hence, the generic result is that $w$ is close to -0.77 today (close to the value derived in tracker models of quintessence) [23] and decreasing towards negative value.

One may argue that the universe at the origin of time is dominated by phantom energy which may be responsible of the initial BANG. The net effect of the phantom energy on the scale factor is to decelerate it with $w<-1$ accompanied with a small cosmological constant. If $w<-1$ persists, then the fate of the universe is quite fantastic and completely different from the possibilities previously discussed in the literature. For phantom energy, the density grows in the future. By reinstating the universe to the same "phantom state" before each big crunch, the acceleration assures that the cycle can repeat and that the cyclic solution is an attractor. This scenario may address the cosmic singularity and therefore may be related to string theory, or more generally, to quantum gravity [24]. In what follows, we dress the following table characterizing the properties of the scaling solutions of the dark energy (present) epoch for different values of $\gamma$ :

TABLE 2

\begin{tabular}{|l|l|l|l|l|l|l|l|l|l|}
\hline$w=\frac{2+p}{3 m}-1$ & $\gamma$ & $p$ & $m$ & $\Lambda=3 \gamma H^{2}$ & $\rho \propto t^{-2-p}$ & $G \propto t^{p}$ & $a \propto t^{m}$ & $\Omega_{m}$ & $\Omega_{\Lambda}$ \\
\hline $\begin{array}{l}\text { Phantom Energy Dominance } \\
w=-10 ; 2+p=-27 m\end{array}$ & 0.036 & 0.0746 & -0.076 & $0.108 H^{2}$ & $t^{-2.0746}$ & $t^{0.0746}$ & $t^{-0.0768}$ & 0.94 & 0.06 \\
\hline $\begin{array}{l}\text { Phantom Energy Dominance } \\
w=-3 ; 2+p=-6 m\end{array}$ & 0.153 & 0.36 & -0.39 & $0.459 H^{2}$ & $t^{-2.36}$ & $t^{0.36}$ & $t^{-0.39}$ & 0.85 & 0.15 \\
\hline $\begin{array}{l}\text { Phantom Energy Dominance } \\
w=-5 / 2 ; 2+p=-9 m / 2\end{array}$ & 0.198 & 0.49 & -0.55 & $0.594 H^{2}$ & $t^{-2.49}$ & $t^{0.49}$ & $t^{-0.55}$ & 0.8 & 0.2 \\
\hline $\begin{array}{l}\text { Phantom Energy Dominance } \\
w=-2 ; 2+p=-3 m\end{array}$ & 0.28 & 0.77 & -0.92 & $0.84 H^{2}$ & $t^{-2.77}$ & $t^{0.77}$ & $t^{-0.923}$ & 0.7 & 0.3 \\
\hline $\begin{array}{l}\text { Phantom Energy Dominance } \\
w=-1.5 ; 2+p=-3 m / 2\end{array}$ & 0.48 & 1.84 & -2.56 & $1.44 H^{2}$ & $t^{-3.84}$ & $t^{1.84}$ & $t^{-2.56}$ & 0.51 & 0.49 \\
\hline $\begin{array}{l}\text { Phantom Energy Dominance } \\
w=-1.2 ; 2+p=-3 m / 5\end{array}$ & 0.24 & 0.63 & 4.38 & $0.72 H^{2}$ & $t^{-2.63}$ & $t^{0.63}$ & $t^{-4.38}$ & 0.75 & 0.25 \\
\hline $\begin{array}{l}\text { Dark Energy Dominance } \\
w \approx-0.77 ; 2+p=0.69 m\end{array}$ & 0.75 & 6 & 12 & $2.1 H^{2}$ & $t^{-8}$ & $t^{6}$ & $t^{12}$ & 0.25 & 0.75 \\
\hline $\begin{array}{l}\text { Matter-Dominated Epoch } \\
w=0 ; 2+p=3 m\end{array}$ & 0.28 & 0.77 & 0.916 & $0.84 H^{2}$ & $t^{-2.77}$ & $t^{0.77}$ & $t^{0.916}$ & 0.7 & 0.3 \\
\hline $\begin{array}{l}\text { Radiation-Dominated Epoch } \\
w=1 / 3 ; 2+p=4 m\end{array}$ & 0.22 & 0.56 & 0.638 & $0.66 H^{2}$ & $t^{-2.56}$ & $t^{0.56}$ & $t^{0.638}$ & 0.77 & 0.23 \\
\hline $\begin{array}{l}\text { Stiff Fluid-Era } \\
w=1 ; 2+p=6 m\end{array}$ & 0.153 & 0.36 & 0.39 & $0.459 H^{2}$ & $t^{-2.36}$ & $t^{0.36}$ & $t^{0.39}$ & 0.85 & 0.14 \\
\hline
\end{tabular}

It is noticeable that the more vacuum energy we have, the more acceleration takes place. In summary, up to this point, we have analyzed the features of the decaying matter density law $\rho \propto \dot{G}^{2} / G^{3}$ motivated from Arnowitt-Deser-Misner (ADM) renormalizable group approach. We have found that, if a flat universe is assumed, a cosmological model with $\rho \propto \dot{G}^{2} / G^{3}, \Lambda=3 \gamma H^{2}$ and positive values of $\dot{G}_{0} / G_{0}$, can reproduce the observational data only if $0.6<\gamma<0.9$ without modifying the General Theory of Relativity or implementing additional curvature invariant terms such as Gauss-Bonnet term $[20,22]$. The universe in the constructed model expands with different constant power-law way and therefore don't trouble the primeval nucleosynthesis and the structure formation set-up. Further, a transition from inflation to deceleration to acceleration is realized, in agreement with supernovae (SN) observations (Gold 182 and SNLS data samples). This 
acceleration has been attributed to a dark energy component with negative pressure which can induce repulsive gravity. The universe is too old and comparison of the numerical results shows that the cosmological constant (vacuum energy) dominates lately. It is noteworthy that accelerated expansion of the universe for the present model may occur in the absence of the cosmological constant. It is worth-mentioning that the fact that the $\Lambda \mathrm{CDM}$ model fits the observations does not necessarily imply the physical existence of dark energy with negative equation of state parameter. If for instance $\gamma=0(\Lambda=0)$ then two independent cases occur: $p= \pm m$. The upper sign gives easily $p=m=2 /(3 w+2)$ and therefore accelerated expansion occurs for $-2 / 3<w<0$ while the lower sign gives straightforwardly $p=-m=-2 /(3 w+4)$ for which acceleration occurs for $-3 / 4<w<-2 / 3$. We summarize some numerical results in the following tables (III and IV):

TABLE 3

\begin{tabular}{|c|c|c|c|c|}
\hline$\Lambda=0 ; p=m=\frac{2}{3 w+2}$ & $p=m$ & $\rho \propto t^{-2-p}$ & $G \propto t^{p}$ & $a \propto t^{m}$ \\
\hline $\begin{array}{c}\text { Dark Energy Dominance } \\
w=-0.5 ;-2 / 3<w<0\end{array}$ & 4 & $t^{-6}$ & $t^{4}$ & $t^{4}$ \\
\hline $\begin{array}{c}\text { Matter-Dominated Epoch } \\
w=0\end{array}$ & 1 & $t^{-3}$ & $t$ & $t$ \\
\hline $\begin{array}{c}\text { Radiation-Dominated Epoch } \\
w=1 / 3\end{array}$ & $2 / 3$ & $t^{-2.66}$ & $t^{2 / 3}$ & $t^{2 / 3}$ \\
\hline $\begin{array}{c}\text { Stiff Fluid-Era } \\
w=1\end{array}$ & $2 / 5$ & $t^{-2.4}$ & $t^{2 / 5}$ & $t^{2 / 5}$ \\
\hline
\end{tabular}

TABLE 4

\begin{tabular}{|c|c|c|c|c|}
\hline$\Lambda=0 ; p=-m=-\frac{2}{3 w+4}$ & $p=-m$ & $\rho \propto t^{-2-p}$ & $G \propto t^{p}$ & $a \propto t^{m}$ \\
\hline $\begin{array}{c}\text { Dark Energy Dominance } \\
w=-0.7 ;-3 / 4<w<-2 / 3\end{array}$ & -1.052 & $t^{-3.052}$ & $t^{-1.052}$ & $t^{1.052}$ \\
\hline $\begin{array}{c}\text { Matter-Dominated Epoch } \\
w=0\end{array}$ & $-1 / 2$ & $t^{-2.5}$ & $t^{-1 / 2}$ & $t^{1 / 2}$ \\
\hline $\begin{array}{c}\text { Radiation-Dominated Epoch } \\
w=1 / 3\end{array}$ & $-2 / 5$ & $t^{-2.4}$ & $t^{-2 / 5}$ & $t^{2 / 5}$ \\
\hline $\begin{array}{c}\text { Stiff Fluid-Era } \\
w=1\end{array}$ & $-2 / 7$ & $t^{-2.28}$ & $t^{-2 / 7}$ & $t^{2 / 7}$ \\
\hline
\end{tabular}

The present day variation of the gravitational coupling constant for both cases is $\left|\dot{G}_{0} / G_{0}\right|=|p / m| H_{0}=H_{0}$. Notice that for the lower case $p=-m$, the product $a(t) G(t)=$ constant. One may attribute the accelerated expansion of the universe for the increasing of the gravitational coupling constant for the upper positive case and to its decreasing with time for the lower negative case. Both cases are interesting from theoretical point of view, i.e. checking the condition for the speeding up expansion it is found that both of the above ranges can accommodate accelerated expansion. In fact, the arguments given here are valid for a varying equation of state, which can change from some value in the past, to a value not equal to -1 today. In case it stabilizes in the future at the value -1 , then the scale factor of the universe may evolve differently, i.e. contracting.

In returns to the previous case, normally the transition from deceleration to acceleration occurs at redshift [25] $z_{q}=[-(1+$ $\left.3 w) \Omega_{\Lambda} / \Omega_{m}\right]^{-1 / 3 w}-1$ which depends on the relation between the matter and the vacuum. This value is too close to the one derived in theQCDMmodel.[22] The dark energy begins to dominate $\rho_{\Lambda} \geq \rho_{m}$ when $z_{D E}=\left[\Omega_{\Lambda} / \Omega_{m}\right]^{-1 / 3 w}-1$. The epoch of the dark energy density domination starts at this redshift. We summarize some numerical results of our model in Table V:

TABLE 5

\begin{tabular}{|c|c|c|}
\hline $\begin{array}{c}\text { Dark Energy Dominance } \\
w \approx-0.77\end{array}$ & $z_{q}=\left[-(1+3 w) \frac{\Omega_{\Lambda}}{\Omega_{m}}\right]^{-\frac{1}{3 w}}-1$ & $z_{D E}=\left[\frac{\Omega_{\Lambda}}{\Omega_{m}}\right]^{-\frac{1}{3 w}}-1$ \\
\hline$\gamma=0.75$ & 0.8 & 0.6 \\
\hline$\gamma=0.65$ & 0.49 & 0.33 \\
\hline$\gamma=0.85$ & 1.36 & 1.1 \\
\hline
\end{tabular}

When combining SN with the measurements of the ratio between the comoving distance to the last scattering surface and the SDSS+2dfGRS BAO distance scale $S_{k} / D_{V}$, it was recently shown that at $95.4 \%$ confidence level that $z_{q}=0.84_{-0.17}^{+0.13}$ for $S_{k} / D_{V}+$ Gold 182 and $z_{q}=0.88_{-0.10}^{+0.12}$ for $S_{k} / D_{V}+$ SNLS. [26] It is noticeable that the values of $z_{q}$ at $\gamma=0.75$ is in better agreement with observations than for $\gamma=0.65$ and $\gamma=0.85$. These solutions are difficult to test observationally for two main reasons: first objects of fixed size like spherical galaxies do not possess sharp edges used in practice for measuring angular size and consequently one has rather to measure isophotal diameters, while objects with well-defined linear dimensions such as double radio sources, are highly dynamical and as a result their intrinsic size is unknown. Therefore, these neoclassical tests are in fact difficult to use in practice. A natural question which arises is whether the observational results on $\Omega_{m}$, $\Omega_{\Lambda}$ and $\dot{G}_{p} / G_{p}$ are solid enough to draw any definite conclusions. Anyway, the situation may improve when additional astronomical observations are obtained in the future, e.g. SNAP missions to give more accurate bounds on $\Omega_{m}, \Omega_{\Lambda}$ and $\dot{G}_{p} / G_{p}$. These findings have to be compared with current observations and further numerical simulations will be further discussed in 
a subsequent work. There remains the important challenge of explaining the arrow of time since in our framework, the universe may be cyclic. Finally, the dark energy model discussed through this work unifies in a natural way the early-time inflation and late-time acceleration whatever (quintessence, cosmological constant or phantom energy) nature they have. However, in order to check if such unification is realistic one, it is necessary to analyze the details of inflation, especially, the preheating scenario. This lies beyond the scopes of our paper. However, the existing astronomical data cannot validate/refuse the cyclic cosmological model discussed here at high confidence level so one awaits the next generation observational data which will be able to reconstruct the realistic and sensible evolution of the cosmological parameters.

Finally, we would like to stress that the constraint $0<\gamma<1$ (positive and tiny cosmological constant) may be obtained if we examine the entropy bound of the dark energy, i.e. the bound we obtain can be interpreted as the bound of the structure of the dark energy observed presently. For this, we fol- low the cosmological arguments of [27] employing the idea of Bekenstein holography: the entropy $S=\sigma L_{h}^{3} / a^{3}(\sigma$ is the entropy density measured in the comoving space, which is constant in time) cannot exceed one unit per Planckian area $A=L_{h}^{2}$ of it boundary's surface characterized by the future event horizon $L_{h}=a(t) \int_{t}^{\infty} d t^{\prime} / a\left(t^{\prime}\right)=t /(m-1)$ and therefore $S / A \approx \sigma /(m-1) t^{(\gamma+1) /(\gamma-1)}$. Combining the requirement that the ratio $S / A$ not increasing with time, positive $L_{h}$ and accelerated expansion $m>1$, we obtain easily $0<\gamma<1$ which is a reasonable constraint for the bound of the cosmological constant, a consequence of the special decaying law $\rho \propto \dot{G}^{2} / G^{3}$ we have supposed. Comparing with the investigation of the observational constraint, we found that this decaying law plays a powerful role in the study of modern cosmology. Finally, it is noteworthy that the main reason for using the future event horizon in place of the particle horizon is that this later is related to the early universe, when dark energy played no role.
[1] A.G. Riess, et al., Astron. J., 116, 1009 (1998).

[2] S. Perlmutter, et al., Astrophys. J., 517, 565 (1999).

[3] B.R. Schmidt, et al., Astrophys. J., 507, 46 (1998).

[4] P.J. Steinhardt, L. Wang and I. Zlatev, Phys. Rev. D, 59 123504(1999).

[5] M. Persic, P. Salucci and F. Stel, Mon. Not. Roy. Astron. Soc., 281, 27 (1996).

[6] M. Carmeli and T. Kuzmenko, Int. J. Theor. Phys., 41, 131( 2002).

[7] S. Behar and M. Carmeli, Int. J. Theor. Phys.. 39, 1375 (2002).

[8] B.P. Schmidt, et al., Astrophys. J., 507, 46 (1998) .

[9] A.G. Riess, et al., Astrophys. J., 607, 665 (2004).

[10] J.S. Alcaniz, Phys. Rev., D69, 083521 (2004).

[11] P. de Bernardis, Nature, 377, 600 (2000).

[12] P.J.E. Peebles and B. Ratra, Rev. Mod. Phys., 75, 559 (2003).

[13] P.h. Brax and Martin, J. Phys. Lett., B468, 45 (1999).

[14] C. Armendariz-Picon, V. Mukhanov and P.J. Steinhardt, Phys. Rev. D63, 103510 (2001).

[15] J.C. Fabris , S.V.B. Concalves and de R. Sa Ribeiro, Gen. Rel. Grav. 38, 495 (2006).

[16] A. Yu Kamenshchik, V. Moschella and V. Pasquier, Phys. Lett., B511, 265 (2001).

[17] J.C. Fabris, S.V.B. Concalves and P.E. de Souza, Gen. Rel. Grav. 34, 53 (2002).

[18] N. Bilic, G.B. Tupper and R.D. Viollier, Phys. Lett. B535, 17 (2002).

[19] See I.A. Arbab astro-ph/0212565 and references therein.

[20] A.R. El-Nabulsi, Mod. Phys. Lett., A23, No. 6, 401 and references therein (2008).
A.R. El-Nabulsi, Chin. Phys. Lett., 25, No. 8, 2785 (2008) and references therein

A.R. El-Nabulsi, Int. J. Mod. Phys. D, (in press)(2008)

[21] A. Bonanno, G. Esposito and C. Rubano, Class. Quantum Grav. 21, 5005 (2004)

A. Bonanno and M. Reuter, Phys. Rev., D65, 043508 (2002)

A. Bonanno and M. Reuter, Phys. Lett., 527B, 9( 2002)

E. Bentivegna, A. Bonanno and M. Reuter, JCAP, 0401:001 (2004)

I.L. Shapiro, J. Sola, C. Espana-Bonet and P. Ruiz-Lapuente, Phys. Lett., 574B, 149 (2003)

J.A. Belinchon, T. Harko and K. Mak, Class. Quantum Grav., 19, 3003 (2002)

B. Guberina, R. Horvat and H. Stefancic, Phys. Rev., D67, 083001 (2003).

[22] A.K. Sanyal, astro-ph/0608104, and references therein

[23] I. Zlatev, L. Wang and P.J. Steinhardt, Phys. Rev. D59, 123504 (1999).

[24] P.J. Steinhardt and N. Turok, Phys. Rev., D65, 126003 and references therein (2002).

[25] O. Sergijenko, and B. Novosyadlyj, astro-ph/0805.3782 and references therein.

[26] E.E. Ishida, R.R.R. Reis, Toribio A V and Waga I astro$p h / 0706.0546$ and references therein

[27] B. Wang, E. Abdalla and R.K. Su, Phys. Lett., B611, 21 and references therein (2005). 